\title{
Proyecto Promover: Attempts to Roll Out an HIV Prevention and Testing Initiative Within a Mexican Immigrant Community
}

\author{
Susan Ryerson Espino ${ }^{1,2}\left[\right.$ (]) Patricia Aguado ${ }^{2,3} \cdot$ Monica Puente ${ }^{2} \cdot$ Pamela Vergara-Rodriguez $^{2,4}$
}

(c) The Author(s) 2020

\begin{abstract}
Latinx immigrants and men, in particular, living in the US are disproportionally impacted by HIV. Despite these concerns, there is limited research on the development, implementation, and evaluation of community-based HIV education and HIV testing interventions. The current study describes such efforts within a historic Mexican immigrant enclave in Chicago. A mixed-methods case study was used to describe intervention development, as well as preliminary evaluation data. Community intervention components were refined through early focus groups, asset mapping, community networking and consultation with cultural advisors. We exceeded our activity goals. We were successful in reaching a segment of the population that is often overlooked and remains unaware of HIV and its risks. Incorporating social network approaches could facilitate reaching at-risk community groups. Demonstration projects require more time and resources (fiscal and technical) to develop, refine, evaluate and sustain community-level intervention components.
\end{abstract}

Keywords Mexican $\cdot$ Latinx $\cdot$ HIV $\cdot$ Intervention development $\cdot$ Community education $\cdot$ Testing

\section{Background}

While we have a vast array of HIV prevention, outreach, and treatment services, there continues to be a need for more sociocultural community education and community-based testing initiatives, as well as, evaluation of the development, implementation, and outcomes of such initiatives [1-4]. To reduce new infections and delays in testing and care, HIV partners need to move outside of their clinic settings into communities at risk to raise awareness, reduce HIV stigma, and normalize HIV testing as a routine aspect of annual health screening $[2,3]$.

Recent CDC data show an overall decline in the rates of HIV infection [5, 6]. However, between 2010 and 2016

Susan Ryerson Espino

srespino@gmail.com

Evaluation Consultant, Chicago, IL, USA

2 The Ruth M. Rothstein CORE Center, Hektoen Institute of Medicine, Chicago, IL, USA

3 Department of Social Work, Northeastern Illinois University, Chicago, IL, USA

4 Cook County Bureau of Health Services, The Ruth M. Rothstein CORE Center, Chicago, IL, USA there was an overall 14\% increase of HIV infections among Latinxs, a 30\% increase among Latinx MSM, and a $68 \%$ increase among Latinx MSM ages 25-34. In 2016, Latinxs accounted for $26 \%$ of the new HIV diagnoses in the United States and 6 dependent areas [5].

The CORE Center's Proyecto Promover was developed as part of a 5-year grant through the Health Resources and Services Administration, Special Projects of National Significance (HRSA-SPNS) to develop, implement and evaluate a multilevel intervention aimed at identifying and engaging Mexican immigrants in HIV care. The goals of our community work included decreasing individual and community HIV-related stigma, normalizing HIV testing and facilitating timely linkage to care for any community member living with HIV.

The current case study describes the development of community intervention components: a testing promotion campaign, community education sessions and community-based testing as well as evaluation methods and findings. We aim to shed light on the work needed to develop new interventions and help others anticipate resources, challenges, and strategies for program development and implementation. 


\section{Conceptual Framework}

From project inception, multisite and local evaluators encouraged a developmental perspective aimed at refining the intervention as well as developing the capacity to implement and evaluate. A descriptive mixed-methods case study approach with collaborative inquiry were used to document and reflect on the evolution of Proyecto Promover and for the purposes of this paper the development, implementation, and evaluation of the project's community intervention efforts [7-9].

\section{Methods}

\section{Participants}

The principal investigator, program director, project staff, local evaluator, and qualitative multisite evaluators worked collaboratively to document and reflect on the development, implementation, and evaluation of Proyecto Promover.

\section{Data Collection}

We examined program archives, including the grant narrative, reports, grant site visit notes, an evolving intervention manual, presentations, communication records, and process notes. Further, monitoring tools, surveys, and process notes were used to monitor and evaluate community intervention activities. An ACCESS database was developed, with the help of an external consultant, to help track partnership data (kind of partner, contact dates, commitment interests vs. actual commitments including agreeing to post testing campaign materials, host educational sessions, or host testing event); actual community education and/or HIV testing sessions (kind of venue, date, participant demographics, number tested, participant-level risk factors, testing history and results).

\section{Measures}

The framework for the case study grew out of an appreciation for the ecosystems perspective on community health interventions and implementation science (see Fig. 1) and focused attention on context and history, the people involved in the intervention, intervention content and methodology, the resource context, and evaluation activities including integration and sustainability of efforts, as well as challenges within the community and organizational context [10-12].

\section{Analysis}

Qualitative content analysis was used to consolidate and summarize highlights relative to framework domains and detail the developmental history of this intervention. Dialogue between the program partners and the evaluator was also used to identify details not explicit in program archives. The evaluator used IBM SPSS version 22 to descriptively analyze exports from ACCESS including outputs and outcomes relating to testing promotion campaign and testing as well as community partnerships and education.

Fig. 1 Case study framework

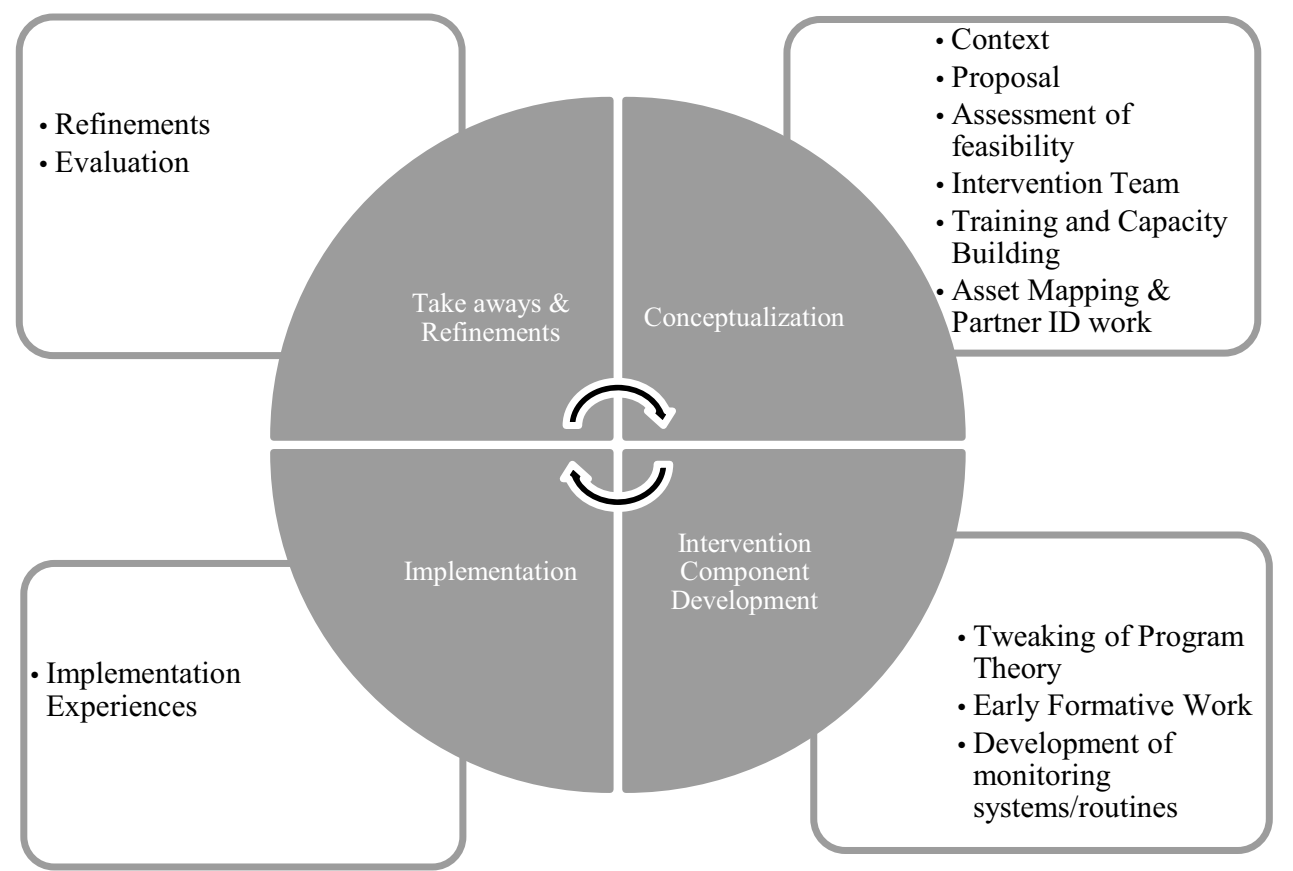


The study protocol was approved by the Institutional Review Board of Cook County Health and Hospitals Systems. Inclusion criteria in community intervention activities included, individuals 18 years of age or older. Those participating in testing and linkage activities signed informed consents. Care was used to maintain confidentiality with password-protected referral and data files. Consents were waived for other activities and no identifiers were stored.

\section{Results}

\section{Community Context}

Proyecto Promover identified Pilsen and Little Village as the neighborhoods within which to launch community interventions due to the high concentrations of Mexican foreign-born community members and the proximity to The CORE Center ( $1 \& 3$ miles). Pilsen and Little Village are in Chicago's Lower West Side and South Lawndale community areas, respectively. The Lower West Side community area has a population of almost 33,000, of whom $76 \%$ are Latino, $30 \%$ are foreign-born Mexicans [13, 14]. The South Lawndale community area has a population of almost 75,000, of whom $83 \%$ are Latino, $36 \%$ are foreign-born Mexicans [13, 14]. There had been no previous intentional efforts by The CORE Center to increase testing or prevention within these communities.

\section{Intervention Team}

The team was led by bilingual, Mexican American providers with over a decade of clinical experience and research with Spanish speaking, Latinx communities living with HIV. The primary interventionist, a community health worker (promotora), was bilingual, bicultural, born and raised in Chicago to immigrant Mexican parents, grew up in the intervention neighborhoods, was affected personally through family experience with HIV, and committed to her community.

The promotora participated in a variety of trainings in preparation for her role as community tester, educator, and outreach worker (trainings summarized in Table 1). Content of trainings reinforced knowledge of infectious diseases and care (HIV, Hepatitis), knowledge and practices relating to testing, and strategies to identify and engage hard to reach patients. The promotora participated in weekly formal supervision with the project director, impromptu discussions throughout the week with colleagues, and routine project meetings. The focus of supervision was to discuss the logistics associated with community intervention development and implementation Supervision also facilitated processing the intrapersonal feelings that emerged while engaged in this work.

Multiple groups consulted with Proyecto Promover including CORE consumers, staff, HIV providers, local and multisite evaluators, a Latino social marketing firm and CCHHS public relations/branding specialists. A cultural advisory board was convened composed of academics, researchers and practitioners in either the HIV and/or Latinx health fields. These groups were instrumental in the early formative work described below.

\section{Early Formative Work}

Our formative work included focus groups, asset mapping and networking, and engaging with cultural advisors to foster further intervention development and grounding within the sociocultural context.

Table 1 Promotora training

\begin{tabular}{ll}
\hline Training topics & Specific trainings \\
\hline Infectious disease knowledge building & Current approaches to HIV care: \\
Testing knowledge and practice & Hepatitis C: management, treatment strategies \& new drugs for a cure \\
& Uni-gold rapid training: Utilization of the uni-gold testing kit \\
& HIV test counseling and HIV test counseling practicum: \\
Strategies and practice: identification \& engagement of at risk commu- & Tips, tricks, \& trends in testing: \\
nity members & HIV and post traumatic stress disorder: \\
& Adherence counseling: \\
& Integrating 12-step recovery with harm reduction in the treatment of \\
& HIV-infected addicts (lecture): \\
& Why can't they just show up? Retention \\
& Street and community outreach intervention: \\
& Get ready, get set, PrEP!: \\
& Motivational interviewing
\end{tabular}




\section{Focus Groups}

Project staff collaborated with a medical provider at The CORE Center to facilitate six focus groups aimed at exploring community HIV knowledge, myths, and barriers to care. Key findings informing the content of our testing promotion campaign and educational sessions included the need to promote that everyone is at risk, testing is preventive care, testing is being responsible for self and loved ones, and that testing was confidential and available at no cost.

\section{Asset Mapping, Networking, and Relationship Building}

The promotora and MSW interns engaged in fieldwork between February 2014 and November 2016 (about 1 day/week). The first four months of fieldwork was spent researching the Pilsen and Little Village communities and walking the community to map assets. This involved documenting community assets that could help promote HIV testing or serve as a site for testing or educational sessions. Paper notes were later entered into a spreadsheet and an intern created a google map to help visualize the community assets. In time, the promotora began networking and relationship building by reaching out to community assets or stakeholders to describe the project and secure partnerships to host educational charlas and/or HIV testing, post our testing promotion campaign, and refer community members living with HIV to The CORE Center. Networking was tracked in the ACCESS database. Throughout the first year of the grant, staff also scoured the internet for HIV testing campaigns and worked on developing project materials with a local Latinx social marketing firm.

\section{Cultural Advisors}

Community members, consumers, cultural advisory board members, staff, county public relations, and evaluation partners provided several rounds of feedback on test promotion and charla content. Relative to campaign materials, edits were made to simplify and focus messaging on testing, refining Spanish messaging, and identifying images representative of community members. Relative to the charlas, cultural advisors challenged the originally conceptualized lecture-style highlighting facts and pressed that the expert/audience format was incongruent with popular education models and adult ways of learning. They encouraged the program to re-conceptualize the charlas away from didactic presentations and toward horizontal conversations where all are participants, all are learning, and all are informing the chat.

\section{Emerging Intervention Content and Mechanisms}

A framework of anchor concepts for the intervention began to emerge that involved elements of evidenced-based health education best practices and tenants from adult [15] and popular education [16]. Concepts included: (1) emphasizing a few key knowledge points about HIV concerns for Latinxs and the testing process; (2) normalizing HIV testing by promoting it as an important, regular, health exam to protect their health and that of their loved ones; (3) validating the personal and community struggles and resiliency in coping with the constraints and resources defining transnational Mexican immigrants living in Chicago, and; (4) encouraging personal and social action by building knowledge and commitments.

Table 2 summarizes how these anchoring concepts were woven throughout each of the community intervention components.

\section{Testing Promotion Campaign}

The testing promotion campaign included posters, palm cards, and flyers. To a lesser extent the campaign also included social media: Facebook, Instagram, newspapers and radio programs. Content emphasized the location of free testing and that routine testing is preventive care and taking responsibility for self and loved ones. Images and language specifically aimed to reflect everyday Mexican immigrant community members to emphasize that everyone is at risk (see Fig. 2).

\section{Community Education Charlas}

Program developers moved away from a comprehensive list of knowledge topics and focused on a set of key functional health knowledge points, key skills, and instructional strategies that emphasized collaboration and self-care. The charlas became a safe, culturally-tailored context for discussions of everyday experiences with HIV, health and prevention, transnational lives and complications that can arise in immigrant communities, personal and cultural values, as well as myths and stigma. Community member experiences and priorities provided the foundation for additional conversation. Charlas typically ran for about a 60-90 min and were mainly in Spanish.

\section{Community Testing}

In June 2014 we began to disseminate our testing promotion campaign, conduct HIV testing and charlas. Attempts were made to track, within the ACCESS database, the distribution of campaign materials (kind, time, and place) as well as event-level and participant-level data from testing 


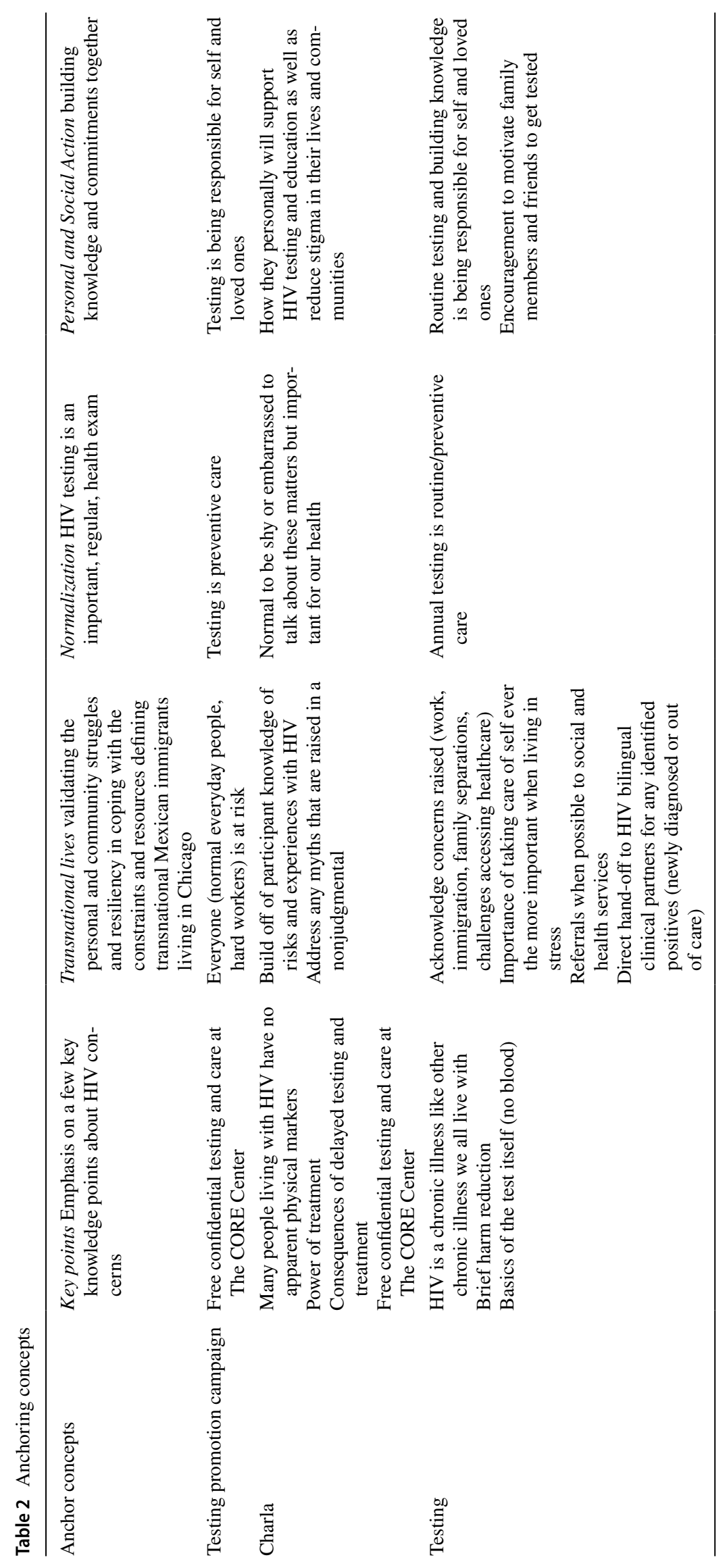




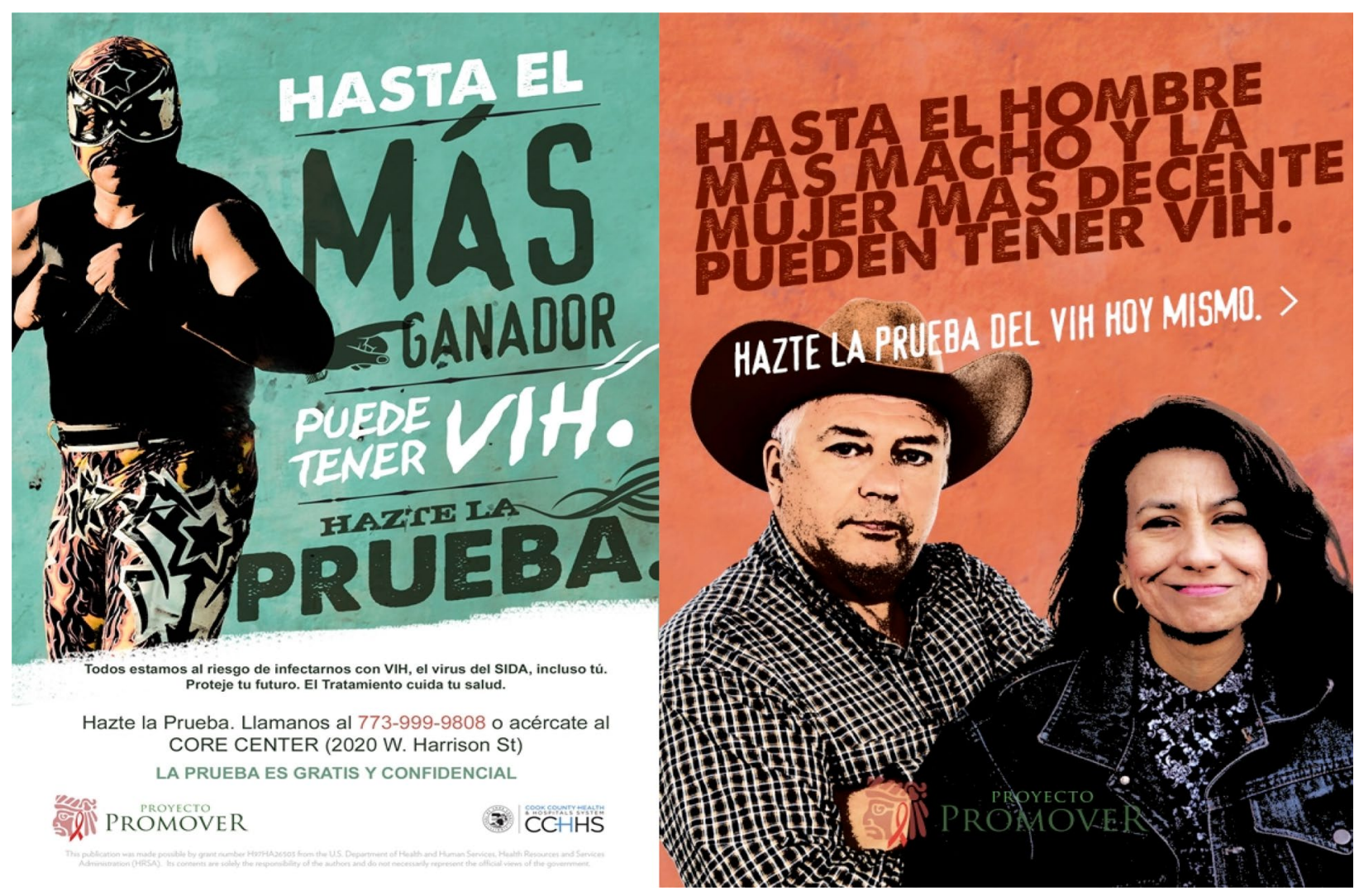

Fig. 2 Testing promotion campaign. The luchador is a popular symbol that transcends the border and reflects the Mexican fighting spirit in Mexico and abroad, Mexican agility, ability to persist and "fight" on against many odds. With the luchador image, we hoped to inspire everyday luchadores to maintain their fight to take care of themselves

and education sessions. Our targets were broad and included: small and large business, health fairs, jornalero (day labor) spots, CBOs, health clinics, botanicas (retail stores with natural and religious remedies), pharmacies, flea markets, churches, substance abuse groups/detox centers, cultural fairs like the Mexican rodeo and Latinx music fests, and the Mexican Consulate of Chicago.

Testers emphasized HIV was a chronic illness that could be effectively treated; the importance of not delaying testing or treatment. They also emphasized annual testing as an aspect of routine health screenings and stressed that annual testing coupled with knowledge of risks and protective behaviors against STDs and HIV, were all important aspects of taking care of self, loved ones, and partners. In the event of a positive test result, the promotora was immediately available to de-brief, provide HIV test post counseling, provide support, and refer to The CORE Center for a confirmatory HIV test. and loved ones. El ranchero y "la virgin" images reflect the many faces we see in and around our communities, within our families, and amongst ourselves. These images depict proud, everyday working people who carry a strong sense of Mexican identity and realities as they navigate life in Chicago

\section{Evaluation: Outputs, Outcomes, and Challenges}

Proyecto Promover exceeded activity goals relating to the distribution of testing promotion campaign materials and the implementation of charlas and testing events (see Table 3). We successfully engaged Spanish-speaking community members, mainly from Mexico, in all community components. Over half of those engaged in testing and charlas were men. On average, participants were young adults but ranged in age between the late teens to the mid '60s.

\section{Social Marketing Campaign}

Intercept surveys were conducted during fall 2015 over the course of two weeks at two busy intersections in the prioritized neighborhoods, during a weekend day and during mid-week evening rush hour. Interviewers invited,in Spanish and English, community members to participate in a brief 
Table 3 Activity outputs

\begin{tabular}{|c|c|}
\hline Component/goals & Outputs \\
\hline Testing promotion campaign & $\begin{array}{l}\text { Distributed } 1735 \text { pieces of social marketing materials; mostly palm cards } \\
\text { followed by posters throughout community and social media posts. } \\
\text { Proyecto Promover and the campaign were also featured in multiple local } \\
\text { and national print and televised media outlets } \\
\text { Conducted intercept survey with } 102 \text { individuals ( } 47 \% \text { male; } 83 \% \text { Latino; } \\
61 \% \text { Mexican) }\end{array}$ \\
\hline $\begin{array}{l}\text { Testing } \\
\text { Goals: } 1000 \text { community tests between September } 2014 \text { and } 2017\end{array}$ & $\begin{array}{l}\text { Conducted } 122 \text { testing events; tested } 1757 \text { individuals (54\% male; 92\% } \\
\text { Latino; 83\% Mexican) }\end{array}$ \\
\hline $\begin{array}{l}\text { Community charlas } \\
\text { Goals: } 3-5 \text { churches and } 3-5 \text { community organizations/business } \\
\text { every year of the } 4 \text { implementation years of the intervention }\end{array}$ & $\begin{array}{l}\text { Established relationships with } 10 \text { churches and } 23 \text { community organizations } \\
1 \text { umbrella hometown association, } \\
4 \text { Chicago civic organizations, } \\
6 \text { health clinics/coalitions, } \\
2 \text { substance abuse treatment centers, } \\
5 \text { community organizations/social service agencies, } \\
3 \text { community fairs, and } \\
2 \text { educational settings } \\
\text { Conducted } 45 \text { sessions across } 33 \text { sites including } 344 \text { community members } \\
\text { (67\% male). The number of group members per group varied but on aver- } \\
\text { age was between } 7 \text { and } 8 \text { participants (range } 5-30)\end{array}$ \\
\hline
\end{tabular}

anonymous survey. The survey elicited data on the frequency of time spent in the prioritized community areas, exposure to the campaign, the impact of the campaign, and engagement in health care. IBM SPSS as used by the evaluator for data entry and descriptive analysis.

Of the 102 community members who participated in intercept surveys, $20 \%$ reported exposure to the print campaign. The majority of community members affirmed the importance of the campaign and the need to promote free and confidential testing. Reflection about risk and testing oneself was reportedly high as a result of seeing the campaign (60 and 65\% respectively for those who had prior exposure and 58 and $64 \%$ for those with no prior exposure). Twenty percent of those reporting prior exposure reported that they were tested as a result of seeing the campaign. Annual testing intentions appeared to be high (70\% of those reporting prior exposure and $77 \%$ of those without prior exposure reported that they would be likely to very likely to have an HIV test once a year after seeing the campaign). Crosstabs were used to examine exposure, reflection about risk, testing, and testing intentions for the largest group of respondents, those Mexican identified. Responses did not vary by country of birth, gender, language preference or age. During the survey, many participants reported that what they really wanted was general health exams but in absence of that, it was good that free confidential testing was available.

\section{Testing}

Proyecto Promover participated in 122 testing events and tested 1757 individuals, including 1451 Mexican and 129 Latinx from other nationalities ( $83 \%$ tested were Mexican;
93\% were Latinx). Testing was highest during summer and early fall months. Over half of those tested reported being first-time testers (56\%). Testing status was found to be dependent on sex and ethnicity. Overall, Mexican participants were least likely to report previous testing overall compared to their counterparts, $40 \%$ of Mexicans reported previous testing compared to $54 \%$ for other Latinx participants and 70\% for non-Latinx participants (Cramers's V, $\mathrm{p}<0.00)$. Women were more likely to report previous HIV testing compared to men $(48.7 \%$ vs. $39.3 \%, \chi 2(1)=15.71$, $\mathrm{p}<0.00)$. Mexican men and women were more often firsttime testers compared to their counterparts from other groups. Only one third (33\%) of those participating in testing reported any sexual risk; multiple partners and unprotected sex were the most commonly reported risk factors ( $26 \%$ and $21 \%$ respectively); a minority reported engaging in sex with other men. Mexican men repeat testers specifically were more likely to report sex with alcohol, multiple partners, condomless sex, and sex with men when compared to Mexican men first-time testers.

\section{Charlas}

Forty-five charlas across 33 sites were delivered including 344 community members. Twenty percent of those who participated in the charlas also completed pre/post surveys (69/344). Knowledge and intentions significantly increased across all measures pre to post with medium effect sizes (0.42-0.65 effect sizes). Post knowledge of local resources was significantly related to prevention knowledge $(r=0.77)$ and intentions to refer a friend or loved one for testing $(r=0.32)$. Prevention knowledge was related to personal 
testing intentions $(r=0.27)$ and intentions to refer a friend or loved one for testing $(r=0.34)$. Intentions to get tested were related to intentions to refer friends or loved ones for testing $(r=0.73)$.

Testing was offered at the end of all charlas. Out of the 45 sessions, testing occurred at 17 or $38 \%$ of the charlas. The confidential setting during HIV testing provided a space for participants to ask questions they were reluctant to raise during group charlas. In addition to getting tested, most individuals in these groups committed to promoting HIV knowledge among friends/family to testing, as well as refer community agencies to program for testing and education events.

Importantly, the promotora shared charla materials and testing strategies developed during Proyecto Promover with another community health organization interested in stepping up education and testing.

\section{Evaluation Challenges}

Lack of time during events (testing and charlas) led to simplifying tools. For instance, a number of forms were used in an attempt to document demographics and risks for those participating in testing. Time constraints limited detailed assessments of risk. Relative to the charlas, the promotora was concerned about using group time for evaluation purposes. We also recognized the need for her to develop comfort in facilitating the groups. Consequently, the use of the pre/post survey was put on hold until year three of the grant. Prior to surveys, the evaluator developed a debriefing form and observation protocol to help monitor strengths and challenges.

There was an initial desire to draft tools to conduct community surveys to assess baseline and post community knowledge of HIV, knowledge of community resources, and stigma. However, the evaluator and program staff were concerned that the community intervention components were of insufficient intensity and spread out over a large neighborhood context to have an impact on these constructs and further, we lacked the resources to do a strong communitywide survey.

\section{Discussion}

While we were unsuccessful in finding many HIV positive individuals through our community efforts, that was not the sole measure of our success. We were successful in reaching a segment of the population that is often overlooked and remains unaware of HIV and its risks. Increased presence and relationship building in neighborhoods that are enclaves for Mexicans or other Latinx groups help inform the creation of programs that are for the people, by of the people. Further, intentionality in the implementation of HIV prevention programming with individuals that may not be deemed highest risk can still be incredibly impactful given the potential to put HIV on the radar of communities who before may have never given HIV more than a passing thought. There are now two Mexican enclaves within the city of Chicago that are further along the spectrum of integrating HIV testing as a routine aspect of good health, a group that has acquired more knowledge not bound in myths or fear, and a subset of people that now know their status because they engaged in HIV testing for the first time in their adult lives.

Future work could include HIV prevention and care promotion through recruitment into charlas and testing events using social network approaches [17, 18]. Social networking approaches involve identifying key recruiters and opinion leaders within high-risk community contexts who could bring in their social network members (through in-person or social media messages) for prevention, testing, and care.

\section{New Contributions to the Literature}

Our efforts, while not novel in nature, confirm the significance of developmental insights in the implementation of culturally-responsive HIV testing interventions. As a field, we need more descriptive accounts of the developmental efforts needed to launch, adapt, and evaluate such interventions. This study offers up a framework for such descriptive accounts informed by work in community health interventions and implementation science. Further, we recommend demonstration projects be allocated sufficient time and resources (fiscal and technical) to develop, refine, evaluate and sustain community-level intervention components. Too often such initiatives come to an end before they have been thoroughly described, reached a stage of full implementation, or adequately evaluated. Employing frameworks like the one described in this article could help us as a field to be more prudent with public resources.

Acknowledgements The authors thank the individuals who participated in this study.

Author Contributions All authors contributed, reviewed and approve the manuscript for submission.

Funding This project was supported by the Health Resources and Services Administration (HRSA) of the U.S. Department of Health and Human Services (HHS) under grant number H97HA26503. The content and conclusions are those of the authors and should not be construed as the official position or policy of, nor should any endorsements be inferred by the HRSA, HHS or U.S. Government.

\section{Compliance with Ethical Standards}

Conflict of interest All authors declare that they have no competing interests. 
Ethical Approval The Institutional Review Board at the Cook County Health and Hospital System in Chicago reviewed and approved this study.

Informed Consent Informed consent was obtained from all individual participants included in the study.

Open Access This article is licensed under a Creative Commons Attribution 4.0 International License, which permits use, sharing, adaptation, distribution and reproduction in any medium or format, as long as you give appropriate credit to the original author(s) and the source, provide a link to the Creative Commons licence, and indicate if changes were made. The images or other third party material in this article are included in the article's Creative Commons licence, unless indicated otherwise in a credit line to the material. If material is not included in the article's Creative Commons licence and your intended use is not permitted by statutory regulation or exceeds the permitted use, you will need to obtain permission directly from the copyright holder. To view a copy of this licence, visit http://creativecommons.org/licenses/by/4.0/.

\section{References}

1. Rios-Ellis B, Becker D, Espinoza L, Nguyen-Rodriguez S, Diaz G, Carricchi A, Galvez G, Garcia M. Evaluation of a community health worker intervention to reduce HIV/AIDS stigma and increase HIV testing among underserved Latinos in the Southwestern US. Public Health Rep. 2015;130(5):458-67.

2. Grieb SMD, Desir F, Flores-Miller A, et al. Qualitative assessment of HIV prevention challenges and opportunities among Latino immigrant men in a new receiving city. J Immigr Minor Health. 2013;17(1):118-24.

3. Penman-Aguilar A, Boye K, Liburd L. Background and rationale. MMWR Suppl. 2016;65(1):2-3.

4. Krishnaratne S, Hensen B, Cordes J, Enstone J, Hargreaves JR. Interventions to strengthen the HIV prevention cascade: a systematic review of reviews. Lancet HIV. 2016;3(7):e307-e317317.

5. Centers for Disease Control and Prevention. Estimated HIV incidence and prevalence in the United States, 2010-2016. HIV Surveillance Supplemental Report 2019; 24 (No. 1). https://www.cdc. gov/hiv/library/reports/hiv-surveillance.html. Accessed 10 July 2019

6. Chicago Department of Public Health (CDPH). STI/HIV Surveillance Report, 2018. Chicago: City of Chicago; 2018.
7. Yin RK. Case study research and applications: design and methods. Thousand Oaks: Sage publications; 2017.

8. Heron J. Co-operative inquiry: research into the human condition. Thousand Oaks: Sage; 1996.

9. Heron J, Reason P. Extending epistemology within a co-operative inquiry. In: Bradbury R, Reason P, editors. The Sage handbook of action research: participative inquiry and practice. Thousand Oaks: Sage Publications; 2008. p. 366-380.

10. Bernal G, Jiménez-Chafey MI, Domenech Rodríguez MM. Cultural adaptation of treatments: a resource for considering culture in evidence-based practice. Prof Psychol Res Pract. 2009;40(4):361.

11. Meyers DC, Durlak JA, Wandersman A. The quality implementation framework: a synthesis of critical steps in the implementation process. Am J Community Psychol. 2012;50(3-4):462-80.

12. Wandersman A, Duffy J, Flaspohler P, Noonan R, Lubell K, Stillman L, Blachman M, Dunville R, Saul J. Bridging the gap between prevention research and practice: the interactive systems framework for dissemination and implementation. Am J of Community Psychol. 2008;41(3-4):171-81.

13. United States Census Bureau/American FactFinder. 2013-2017 American Community Survey 5-Year Estimates. U.S. Census Bureau's American Community Survey Office, 2017. Accessed August 2019

14. Migration Policy Institute tabulation of data from the U.S. Census Bureau's pooled 2013-2017 American Community Survey. Accessed July 32019

15. Knowles MS, Holton EF III, Swanson RA. The adult learner. Abingdon: Routledge; 2012.

16. Freire P. Pedagogy of freedom: ethics, democracy, and civic courage. Lanham: Rowman \& Littlefield Publishers; 2000.

17. Emerson C, Brown T, Illemsky S. Use of social networks to identify persons with undiagnosed HIV infection-seven U.S. cities, October 2003-September 2004. MMWR Morb Mortal Wkly Rep. 2005;54(24):601-5.

18. Latkin CA, Davey-Rothwell MA, Knowlton AR, Alexander KA, Williams CT, Boodram B. Social network approaches to recruitment, HIV prevention, medical care, and medication adherence. J Acquir Immune Defic Syndr. 2013;63(Suppl 1):S54-S5858.

Publisher's Note Springer Nature remains neutral with regard to jurisdictional claims in published maps and institutional affiliations. 